Pacific Journal of Mathematic 


\section{A CHARACTERIZATION OF REGULAR MAXIMAL IDEALS}

\section{ROBERT WARNer AND Robert Whitley}

In 1967, Gleason characterized the maximal ideals in a commutative Banach algebra $A$ with identity as those subspaces $M$ of codimension one in $A$ which contain no invertible elements. (Kahane and Zelazko gave the same characterization in 1968.) An equivalent statement of this property of $M$ is: (1) Each element of $M$ belongs to some regular maximal ideal.

The question we examine is when does this distinguish the regular maximal ideals from the other subspaces of codimension one in a commutative Banach algebra without identity.

We show that if $A$ is generated by a single element then a closed subspace $M$ of codimension one in $A$ and satisfying (1) is a regular maximal ideal and we show by an example that this result may fail for an algebra which is generated two elements. We have results related to the above, which can be applied to $L^{1}(G)$, where $G$ is a locally compact abelian metrizable group; a sample corollary is that a (not a priori closed) subspace $M$ of codimension one for which (1) holds is a regular maximal ideal in $L^{1}(G)$.

That property (1) fails to characterize regular maximal ideals in an arbitrary commutative Banach algebra can be easily seen by considering a locally compact Hausdorff space $S$ which is not sigmacompact, i.e., which is not a countable union of compact sets, and the Banach algebra $C_{0}(S)$ of continuous functions vanishing at infinity on $S$ taken with the supremum norm. For any $f$ in $C_{0}(S),\{s: f(s) \neq 0\}$ is sigma-compact and so $f$ belongs to infinitely many regular maximal ideals in $C_{0}(S)$. Consequently any subspace $M$ of codimension one in $C_{0}(S)$ has property (1) and yet need not be a regular maximal ideal. This example points out that we should consider only algebras in which some element belongs to no regular maximal ideal.

Lemma 1. Let $A$ be a commutative Banach algebra and $x_{0}$ an element belonging to no regular maximal ideal. Suppose that $M$ is a subspace of codimension one in $A$ with the property that each element in $M$ belongs to some regular maximal ideal and let $x^{*}$ be a nonzero linear functional on $A$, not necessarily continuous, which vanishes (precisely) on $M$. Then for all $a$ and $b$ in $A$,

$$
x^{*}\left(x_{0}\right) x^{*}\left(a b x_{0}\right)=x^{*}\left(a x_{0}\right) x^{*}\left(b x_{0}\right) .
$$

Proof. We adjoin an identity $e$ to $A$ in the usual way [6, p. 59] 
obtaining the Banach algebra $A_{e}$ of pairs $(\alpha, \lambda)$ where $\alpha$ is in $A$ and $\lambda$ is a complex number. Recall that $(a, \lambda)(b, \mu)=(a b+\lambda b+\mu a, \lambda \mu)$, $e=(0,1)$ and $\|(a, \lambda)\|=\|a\|+|\lambda|$.

Define the subspace $M_{0}$ of $A_{e}$ by: $M_{0}=\left\{(a, \lambda):(a, \lambda)\left(x_{0}, 0\right)\right.$ is in $M \times\{0\}\}$. Let $(a, \lambda)$ in $A_{e}$ be given and set $\mu=\lambda+\left(x^{*}\left(a x_{0}\right) / x^{*}\left(x_{0}\right)\right)$, noting that $x^{*}\left(x_{0}\right) \neq 0$ since $x_{0}$ is not in $M$, and consider $(a, \lambda)-\mu e$. Because $x^{*}\left(a x_{0}+(\lambda-\mu) x_{0}\right)=0, a x_{0}+(\lambda-\mu) x_{0}$ is in $M$ and so $A_{e}=$ $M_{0}+s p(e)$.

We now show that $M_{0}$ contains no invertible elements. Suppose that $(a, \lambda)$ is an invertible element in $M_{0}$ with inverse $(b, \mu)$. Then

$$
\begin{gathered}
a b+\lambda b+\mu a=0, \\
\lambda \mu=1, \text { and } \\
a x_{0}+\lambda x_{0} \text { belongs to } M .
\end{gathered}
$$

From (5) there is a point $s$ in the regular maximal ideal space of $A$ with $\hat{a}(s) \hat{x}_{0}(s)+\lambda \hat{x}_{0}(s)=0$ and thus $\hat{a}(s)=-\lambda$. Evaluating expression (3) at $s$ we obtain $\hat{a}(s) \hat{b}(s)+\lambda \hat{b}(s)+\mu \hat{a}(s)=-\lambda \mu=0$, which contradicts (4).

Applying Gleason's characterization [3], $M_{0}$ must be a maximal ideal in $A_{e}$. Let $a$ and $b$ be in $A$ and choose $\lambda=-x^{*}\left(a x_{0}\right) / x^{*}\left(x_{0}\right)$. Then $(a, \lambda)$ is in $M_{0}$ and consequently $(a, \lambda)(b, 0)$ is in $M_{0}$, i.e., $x^{*}\left(a b x_{0}+\lambda b x_{0}\right)=0$ which yields equation (2).

THEOREM 2. Let $A$ be a commutative Banach algebra generated by the element $x_{0}$. If a closed subspace $M$ of codimension one in $A$ has the property that each element in $M$ belongs to some regular maximal ideal then $M$ is a regular maximal ideal.

Proof. Let $z$ and $w$ be in $A$. Since $A x_{0}$ is a dense ideal there is a sequence $\left\{a_{n}\right\}$ with $w=\lim a_{n} x_{0}$. Let $x^{*}$ be a nonzero continuous linear functional on $A$ with $x^{*}(M)=0$. Using equation (2), $x^{*}(z w)=$ $\lim x^{*}\left(a_{n} z x_{0}\right)=\lim x^{*}\left(a_{n} x_{0}\right) x^{*}\left(z x_{0}\right) / x^{*}\left(x_{0}\right)=x^{*}(w) x^{*}\left(z x_{0}\right) / x^{*}\left(x_{0}\right)$. Thus if $w$ is in $M, z w$ must be in $M$.

ExAMPLE 3 . We display a commutative Banach algebra $A$ generated by two elements (and therefore with a sigma-compact regular maximal ideal space) with the property that every element in it belongs to infinitely many regular maximal ideals. For this algebra, any subspace of codimension one has property (1) and so (1) does not characterize regular maximal ideals in $A$. The algebra $A$ further shows that a subspace of codimension one in $A$ having property (1) need not be closed; we ask whether this is possible for an algebra 
with a single generator. For the example let $S$ be the bicylinder, $\{(\lambda, \mu): \lambda$ and $\mu$ complex numbers with $|\lambda| \leqq 1$ and $|\mu| \leqq 1\}$. Let $P$ be the algebra of all polynomials in the two variables $\lambda$ and $\mu$ which vanish at $(0,0)$ and let $A$ be the closure of $\mathrm{P}$ in $C(S) . A$ is generated by $\lambda$ and $\mu$. Any nonzero multiplicative linear function $x^{*}$ on $A$ has norm not exceeding one and so the scalars $x^{*}(\lambda)=\lambda_{0}$ and $x^{*}(\mu)=\mu_{0}$ each have absolute value at most one. Thus the pair $\left(\lambda_{0}, \mu_{0}\right)$ lies in $S-\{(0,0)\}$, and for each $f$ in $A, x^{*}(f)=f\left(\lambda_{0}, \mu_{0}\right)$. That is, the regular maximal ideal space of $A$ can be identified with $S-\{(0,0)\}$. Any function $f$ in $A$ is an analytic function in two complex variables on the interior of $S[2$, Theorem 9.12.1, p. 229] which vanishes at $(0,0)$ and consequently [2, Problem 4, p. 245] vanishes at infinitely many points in $S$. The algebra $A$ is then the desired example.

A commutative Banach algebra $A$ has an approximate identity if there is a generalized sequence $\left\{u_{\alpha}\right\}$ of elements of $A$ with $a=\lim u_{\alpha} a$ for all $a$ in $A$, and has a bounded approximate identity if, in addition, the elements $u_{\alpha}$ are uniformly bounded in norm. This usage is not standard; one usually discusses only a bounded approximate identity which is then called an approximate identity. An interesting Banach algebra having an unbounded approximate identity is the algebra $L^{1}(G) \cap G^{2}(G), G$ a locally compact abelian topological group [9, Definition 4.6 , p. 411]. We point out that one cannot generally modify an unbounded approximate identity to get a bounded one. In fact if a Banach algebra $A$ has an unbounded approximate identity $\left\{u_{n}\right\}$ which is a sequence then it cannot have a bounded approximate identity $\left\{v_{\alpha}\right\}$. To see this, one observes that the operators $T_{n}$ defined $T_{n} x=u_{n} x$ are pointwise convergent. Hence, by the Uniform Boundedness Theorem, $\sup \left\|T_{n}\right\|$ is finite, so that $\left\|u_{n}\right\|=\lim _{\alpha}\left\|T_{n} v_{\alpha}\right\| \leqq \sup \left\|T_{n}\right\| \sup \left\|v_{\alpha}\right\|$.

THeOREM 4. Let $A$ be a commutative Banach algebra with an approximate identity each element of which belongs to no regular maximal ideal, for example $A$ a Banach algebra with an approximate identity, a continuous involution $x \rightarrow \widetilde{x}$ with $\widetilde{x}^{\wedge}=\widetilde{x}^{-}$, and a sigmacompact regular maximal ideal space. Then a closed subspace $M$ of codimension one in $A$ with the property that each element in $M$ belongs to some regular maximal ideal must be a regular maximal ideal.

Proof. First we show that the hypotheses are satisfied by $A$ having an approximate identity $\left\{u_{\alpha}\right\}$, an involution $x \rightarrow \widetilde{x}$, as above, and a sigma-compact regular maximal ideal space. Because the involution is continuous $\left\{\widetilde{u}_{\alpha}\right\}$ is also an approximate identity and the elements $w_{\alpha}=\left(u_{\alpha}+\widetilde{u}_{\alpha}\right) / 2$ form an approximate identity with $\hat{w}_{\alpha}$ realvalued. The algebra $A$ is selfadjoint [6, p. 89] with a sigma-compact regular maximal ideal space and so there is an element $v$ in $A$ with 
$\hat{v}(s)$ strictly greater than zero for each $s$ in $\Delta(A)$. The generalized sequence with entries $v_{\alpha, n}=w_{\alpha}+i v / 2^{n}$ and the usual product ordering forms an approximate identity for $A$ and each $v_{\alpha, n}$ belongs to no regular maximal ideal.

Now suppose that $A$ has an approximate identity $\left\{e_{\alpha}\right\}$, no $e_{\alpha}$ belonging to a regular maximal ideal, and suppose that $x^{*}$ is a nonzero continuous linear functional on $A$ with $x^{*}(M)=0$. Using Lemma 1, for each $a$ and $b$ in $A$ we have

$$
x^{*}\left(e_{\alpha}\right) x^{*}\left(a b e_{\alpha}\right)=x^{*}\left(a e_{\alpha}\right) x^{*}\left(b e_{\alpha}\right) .
$$

By passing to a subnet we may suppose that $\left\{x^{*}\left(e_{\alpha}\right)\right\}$ converges, either to a complex number $\beta$ or to infinity. If $\left\{x^{*}\left(e_{\alpha}\right)\right\}$ converges to infinity, then from (6) we see that $x^{*}(a b)=0$ for all $a$ and $b$ in $A$ and consequently $x^{*}(a)=\lim x^{*}\left(a e_{\alpha}\right)=0$ for each $a$, a contradiction. If $\left\{x^{*}\left(e_{\alpha}\right)\right\}$ converges to $\beta=0$, then from (6) $x^{*}$ is again zero. Thus $\left\{x^{*}\left(e_{\alpha}\right)\right\}$ converges to a nonzero complex number $\beta$. By taking limits in (6) we conclude $x^{*} / \beta$ is a multiplicative linear functional and therefore that $M$ is a regular maximal ideal.

THEOREM 5. Let $A$ be a commutative Banach algebra with a bounded approximate identity $\left\{u_{\alpha}\right\}$ having $\operatorname{Re} \widehat{u}_{\alpha}(s) \supsetneqq 0$ for all $s$ in the regular maximal ideal space of $A$ and all $\alpha$, for example $A$ a semisimple commutative algebra with a bounded approximate identity and a sigma-compact regular maximal ideal space. Then a subspace $M$ of codimension one in $A$, which is not a priori closed, having the property that each element in $M$ belongs to some regular maximal ideal must be a regular maximal ideal.

Proof. To see that a semi-simple Banach algebra $A$ with a bounded approximate identity and a sigma-compact maximal ideal space has an approximate identity of the desired type we use some recent results of D.C. Taylor. Under these circumstances $A$ has a bounded approximate identity $\left\{v_{\alpha}\right\}$ with $\operatorname{Re} \widehat{v}_{\alpha}(s) \geqq 0$ for all $s$ and $\alpha$ [8, Corollary 2.3] and contains an element $v$ with $\operatorname{Re} \hat{v}(s) \supsetneqq 0$ for all $s$ in $\Delta(A)$ [8, Th. 1.1]. The generalized sequence $\left\{v_{\alpha}+v / 2^{n}\right\}$ under the product ordering is an approximate identity of the desired type.

Let $A$ have a bounded approximate identity $\left\{u_{\alpha}\right\}$ as hypothesized and suppose that $x^{*}$ is a nonzero linear functional, not a priori continuous, with $x^{*}(M)=0$. Let $b$ and: $c$ in $A$ be given. By Paul Cohen's factorization theorem [1, Th. 1] and by examining the conclusion of the proof on page 202 it follows that $c=a x_{0}$ where $x_{0}=\sum \gamma(1-\gamma)^{n-1} u_{\alpha_{n}}$, $0<\gamma<\frac{1}{4}$, and each $u_{\alpha_{n}}$ a member of $\left\{u_{\alpha}\right\}$. Since $\operatorname{Re} \hat{x}_{0}(s) \supsetneqq 0$ for all $s$ in $\Delta(A)$ we can apply Lemma 1 to conclude that 


$$
x^{*}\left(x_{0}\right) x^{*}(b c)=x^{*}\left(x_{0}\right) x^{*}\left(a b x_{0}\right)=x^{*}\left(a x_{0}\right) x^{*}\left(b x_{0}\right)=x^{*}(c) x^{*}\left(b x_{0}\right) .
$$

Hence if $c$ is in $M, b c$ is in $M$ and $M$ is a maximal ideal. To complete the proof it will suffice to show that the maximal ideals of $A$ are all regular. Since $A^{2}=A$ [1], this follows from Theorem $4.0 \mathrm{~b}$ and Corollary 4.0.3 in [7, pp. 87-88].

\section{REFERENCES}

1. P. Cohen, Factorization in group algebras, Duke Math. J. 26 (1959), 199-205.

2. J. Dieudonné, Foundations of Modern Analysis, Academic Press, 1960.

3. A. Gleason, A characterization of maximal ideals, J. D'Analyse Math. 19 (1967), 171-172.

4. E. Hille and R. Phillips, Functional Analysis and Semi-Groups, Amer. Math. Soc. Colloq. Pub. Vol. 31, Waverly Press, 1957.

5. J. P. Kahane and W. Zelazko, A characterization of maximal ideals in commutative Banach algebras, Studia Math. 29 (1968), 340-343.

6. L. Loomis, An Introduction to Abstract Harmonic Analysis, Van Nostrand, 1953.

7. P. Porcelli, Linear Spaces of Analytic Functions, Rand McNally, 1966.

8. D. C. Taylor, A generalized Fatou theorem for Banach algebras (to appear in Pacific J. Math.)

9. C. R. Warner, Closed ideals in the group algebra $L^{1}(G) \cap L^{2}(G)$, Trans. Amer. Math. Soc. 121 (1966), 408-423.

Received November 21, 1968. Work of the second author supported by National Science Foundation Grant GP-8291.

UNIVERSITY OF MARYLAND AND

UNIVERSITY OF CALIFORNIA, IRVINE 



\section{PACIFIC JOURNAL OF MATHEMATICS}

\section{EDITORS}

H. ROYDEN

Stanford University

Stanford, California

Richard Pierce

University of Washington

Seattle, Washington 98105
J. DugundJI

Department of Mathematics

University of Southern California

Los Angeles, California 90007

BASIL GORDON

University of California

Los Angeles, California 90024

\section{ASSOCIATE EDITORS}

E. F. BECKENBACH

B. H. NeUmanN

F. WOLF

K. YOSHIDA

\section{SUPPORTING INSTITUTIONS}

UNIVERSITY OF BRITISH COLUMBIA

CALIFORNIA INSTITUTE OF TECHNOLOGY

UNIVERSITY OF CALIFORNIA

MONTANA STATE UNIVERSITY

UNIVERSITY OF NEVADA

NEW MEXICO STATE UNIVERSITY

OREGON STATE UNIVERSITY

UNIVERSITY OF OREGON

OSAKA UNIVERSITY

UNIVERSITY OF SOUTHERN CALIFORNIA
STANFORD UNIVERSITY

UNIVERSITY OF TOKYO

UNIVERSITY OF UTAH

WASHINGTON STATE UNIVERSITY

UNIVERSITY OF WASHINGTON

$* * \quad * \quad *$
AMERICAN MATHEMATICAL SOCIETY
CHEVRON RESEARCH CORPORATION
TRW SYSTEMS
NAVAL WEAPONS CENTER




\section{Pacific Journal of Mathematics}

Vol. 30, No. $1 \quad$ September, 1969

William Wells Adams, Simultaneous diophantine approximations and cubic irrationals ..................................... 1

Heinz Bauer and Herbert Stanley Bear, Jr., The part metric in convex

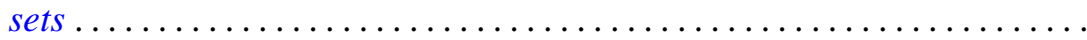

L. Carlitz, A note on exponential sums ...................... 35

Vasily Cateforis, On regular self-injective rings ................. 39

Franz Harpain and Maurice Sion, A representation theorem for measures on infinite dimensional spaces ......................... 47

Richard Earl Hodel, Sum theorems for topological spaces .............. 59

Carl Groos Jockusch, Jr. and Thomas Graham McLaughlin, Countable

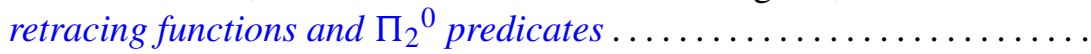

Bjarni Jónsson and George Stephen Monk, Representations of primary

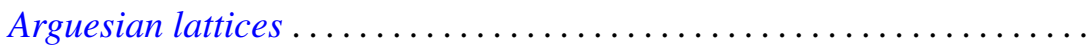

Virginia E. Walsh Knight, A continuous partial order for Peano continua...................................... 141

Kjeld Laursen, Ideal structure in generalized group algebras ........... 155

G. S. Monk, Desargues' law and the representation of primary lattices . . . 175

Hussain Sayid Nur, Singular perturbation of linear partial differential equation with constant coefficients ..........................

Richard Paul Osborne and J. L. Stern, Covering manifolds with cells ... 201

Keith Lowell Phillips and Mitchell Herbert Taibleson, Singular integrals in several variables over a local field...

James Reaves Smith, Local domains with topologically $T$-nilpotent radical....

Donald Platte Squier, Elliptic differential equations with discontinuous coefficients .................................

Tae-il Suh, Algebras formed by the Zorn vector matrix...

Earl J. Taft, Ideals in admissible algebras . .................... 259

Jun Tomiyama, On the tensor products of von Neumann algebras........ 263

David Bertram Wales, Uniqueness of the graph of a rank three group ..... 271

Charles Robert Warner and Robert James Whitley, A characterization of regular maximal ideals ......................... 